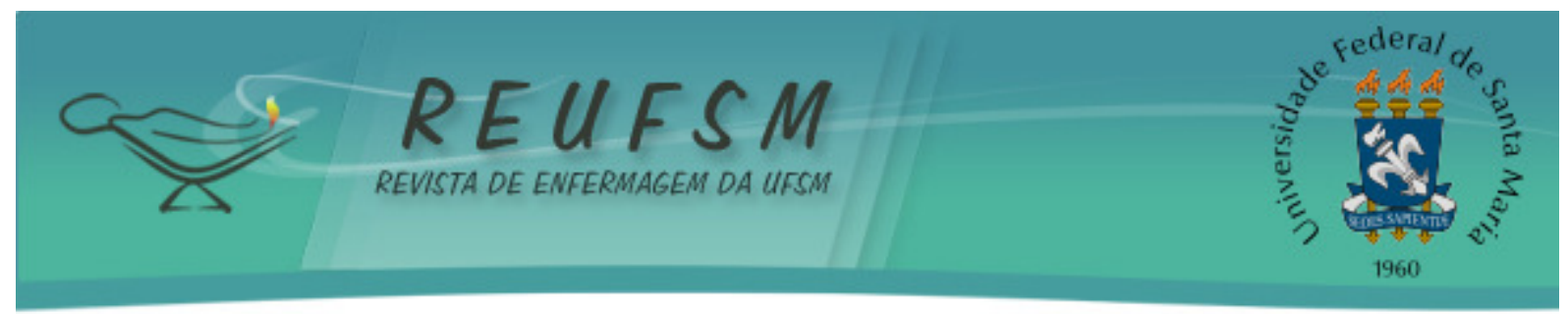

ARTIGO ORIGINAL

\title{
DIAGNÓSTICOS DE ENFERMAGEM MAIS FREQUENTES EM UMA UNIDADE DE TERAPIA INTENSIVA PEDIÁTRICA
}

\section{THE MOST FREQUENT NURSING DIAGNOSIS IN PEDIATRIC INTENSIVE CARE UNIT \\ DIAGNÓSTICOS DE ENFERMERÍA MÁS FRECUENTES EN UNA UNIDAD DE CUIDADOS INTENSIVOS PEDIÁTRICOS}

Doi: $10.5902 / 2179769216471$

Danila Maria Batista Guedes ${ }^{1}$ Lisabelle Mariano Rossato Eloíde André Oliveira ${ }^{3}$

RESUMO: Objetivo: identificar os dez diagnósticos de enfermagem mais frequentes em pacientes internados em uma Unidade de Terapia Intensiva Pediátrica. Método: estudo transversal, com 30 pacientes de uma Unidade de Terapia Intensiva Pediátrica. Os dados foram coletados durante os meses de janeiro e fevereiro de 2013. Resultados: os diagnósticos de enfermagem, segundo a taxonomia II da North American Nursing Diagnosis Association: Risco de infecção $(86,67 \%)$, Motilidade gastrintestinal disfuncional $(60 \%)$, Desobstrução ineficaz de vias aéreas $(53,3 \%)$, Risco de integridade da pele prejudicada (40\%), Risco de trauma vascular (40\%), Risco de desequilíbrio eletrolítico $(36,67 \%)$, Ventilação espontânea prejudicada $(36,67 \%)$, Dor aguda $(33,3 \%)$, Constipação $(33,3 \%)$, Integridade da pele prejudicada $(26,67 \%)$. Conclusão: a identificação dos diagnósticos de enfermagem podem contribuir para implantação da Sistematização da Assistência de Enfermagem na Unidade de Terapia Intensiva Pediátrica, permitindo que a assistência prestada seja direcionada e específica aos reais e potenciais problemas de saúde do sujeito a ser cuidado.

Descritores: Processos de enfermagem; Unidades de terapia intensiva pediátrica; Cuidados de enfermagem.

ABSTRACT: Aim: to identify the ten most frequent nursing diagnosis in hospitalized patients in a Pediatric Intensive Care Unit. Method: cross-sectional study with 30 patients in a Pediatric Intensive Care Unit. The data were collected during the January and February 2013. Results: the nursing diagnosis, according to the taxonomy of North American Nursing Diagnosis Association: Risk of infection (86,67\%), Dysfunctional gastrointestinal motility (60\%), Ineffective airway clearance (53,3\%), Risk of impaired skin integrity (40\%), Risk of vascular trauma (40\%), Risk of electrolyte imbalance $(36,67 \%)$, Impaired spontaneous ventilation (36,67\%), Acute pain $(33,3 \%)$, Constipation $(33,3 \%)$, Impaired skin integrity (26,67\%). Conclusions: the identification of the nursing diagnosis can contribute to implementation of the Nursing Care Systematization at the Pediatric Intensive Care Unit, allowing that assistance to be targeted and specified to actual and potential health problems of the subject to be cared.

Descriptors: Nursing Process; intensive care units, Pediatric; Nursing care.

\footnotetext{
${ }^{1}$ Enfermeira. Mestranda em Ciências pelo Programa de Pós Graduação em Enfermagem da Escola de Enfermagem da Universidade de São Paulo. São Paulo, SP, Brasil. E-mail: danilaguedes@usp.br

${ }^{2}$ Enfermeira. Doutora em Enfermagem pela Universidade de São Paulo. Docente do Departamento de Enfermagem Materno-Infantil e Psiquiátrica da Escola de Enfermagem da Universidade de São Paulo. São Paulo, SP, Brasil. E-mail: rossato@usp.br

${ }^{3}$ Enfermeira. Mestre em Enfermagem pela Universidade Federal da Paraíba. Docente do Curso de Graduação em Enfermagem da Universidade Estadual da Paraíba. João Pessoa, PB, Brasil. E-mail: eloideandre@hotmail.com
} 


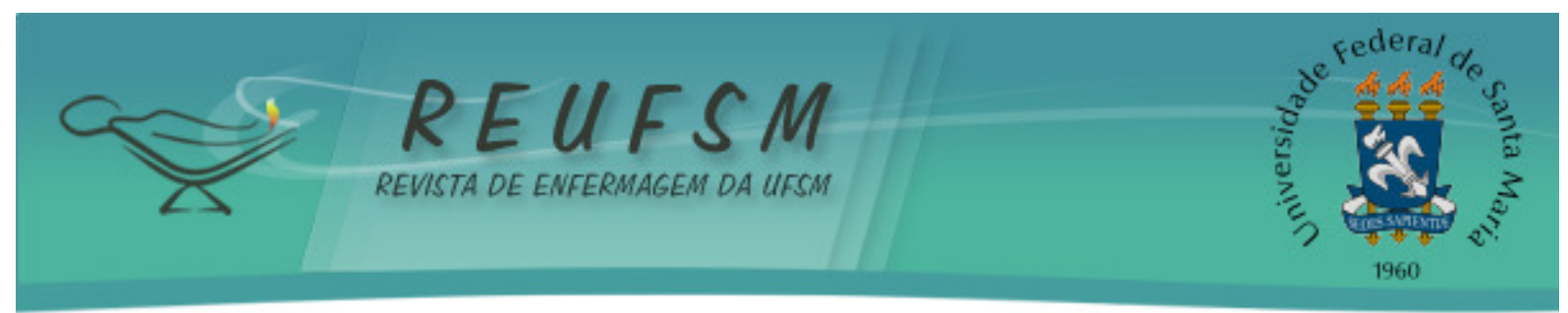

RESUMEN: Objetivo: identificar diez diagnósticos de enfermería más frecuentes en los pacientes hospitalizados en una Unidad de Cuidados Intensivos Pediátricos. Métodos: estudio transversal con 30 pacientes. Los datos fueron recolectados durante enero y febrero de 2013. Resultados: los diagnósticos de enfermería, según la taxonomía II de la North American Nursing Diagnosis Association: Riesgo de infección (86,67\%), La motilidad gastrointestinal disfuncional (60\%), Eliminación de la vía aérea ineficaz $(53,3 \%)$, riesgo de integridad de la piel (40\%), El riesgo de trauma vascular (40\%), Riesgo de desequilibrio electrolítico (36,67\%), Alteración de la ventilación espontánea $(36,67 \%)$, Dolor agudo (33,3\%), Estreñimiento (33,3\%), La integridad de la piel alterada (26,67\%). Conclusión: la identificación del diagnóstico de enfermería puede contribuir a la implementación de la Sistematización de la Asistencia, lo que permite que la ayuda se dirija a los problemas de salud reales y potenciales del sujeto.

Descriptores: Procesos de Enfermería; Unidades de Cuidado Intensivo Pediátrico; Atención de Enfermería.

\section{INTRODUÇÃO}

As Unidades de Terapia Intensiva Neonatal (UTINs) e as Unidades de Terapia Intensiva Pediátrica (UTIPs), foram criadas com o objetivo de salvar a vida de crianças mediante a realização de procedimentos complexos e invasivos aliados à utilização de tecnologias cada vez mais específicas. ${ }^{1}$

0 processo de trabalho de enfermagem em UTI é caracterizado por atividades assistenciais complexas que exigem alta competência técnica e científica, afinal, a tomada de decisões imediatas e adoção de condutas seguras estão diretamente relacionadas à vida e morte de pessoas. Nesse contexto, é de suma importância prover e manter o pessoal de enfermagem qualificado e adequadamente dimensionado para desenvolver a assistência de enfermagem com qualidade e segurança. ${ }^{2}$

A Sistematização da Assistência de Enfermagem (SAE) configura-se como uma metodologia para organizar e sistematizar o cuidado, com base nos princípios do método científico. Tem como objetivos identificar as situações de saúde-doença e as necessidades de cuidados de enfermagem, bem como subsidiar as intervenções de promoção, prevenção, recuperação e reabilitação da saúde do indivíduo, família e comunidade. ${ }^{3}$

A SAE busca promover uma assistência holística, individualizada e mais qualificada ao paciente, consolidando o comprometimento e a responsabilidade do profissional perante os pacientes, familiares e comunidade. Nesse sentido, proporciona ações tanto na área assistencial quanto administrativa, o que facilita a organização do trabalho e dos serviços da equipe de enfermagem. ${ }^{4}$

O processo de enfermagem, considerado a base de sustentação da SAE, é constituído por fases ou etapas que envolvem a identificação de problemas de saúde do cliente, o delineamento do diagnóstico de enfermagem, a instituição de um plano de cuidados, a implementação das ações planejadas e a avaliação. ${ }^{5}$

A segunda fase é o diagnóstico de enfermagem que, segundo a North American Nursing Diagnosis Association (NANDA), é “um julgamento clínico sobre a resposta de um indivíduo, uma família ou uma comunidade com relação a problemas de saúde reais ou potenciais/processos de vida que fornecem a base para uma terapia definitiva, que busca alcançar resultados nos quais a enfermagem é necessária". Cada diagnóstico apresenta componentes estruturais: o título, a definição, os fatores relacionados, fatores de risco e as características definidoras. ${ }^{6}$

Os fatores relacionados são a etiologia do problema, e podem ser de natureza fisiológica, psicológica, sociocultural, ambiental e espiritual. Já os fatores de risco, são os 


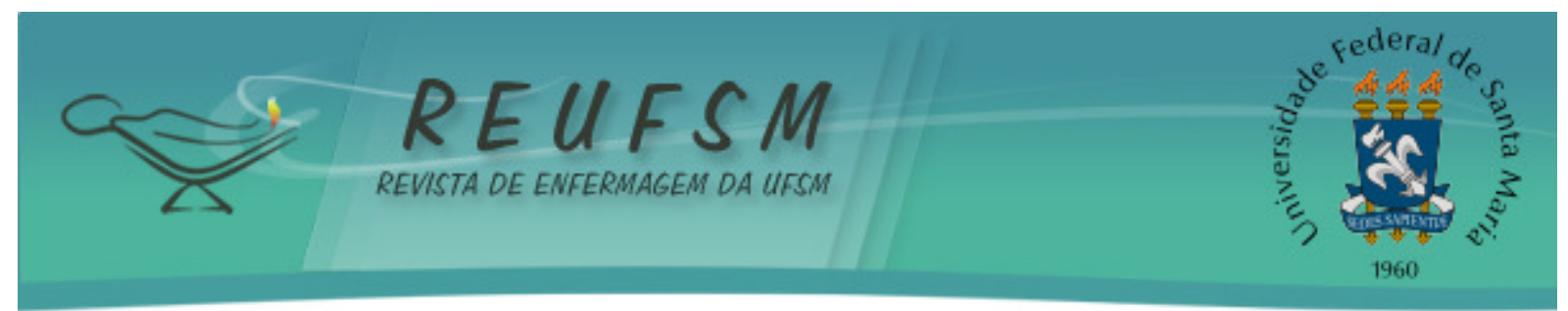

que aumentam a vulnerabilidade de um indivíduo, de uma família, ou de uma comunidade a um evento, sejam eles ambientais, fisiológicos, psicológicos, genéticos ou químicos. As características definidoras são as manifestações clínicas, as evidências que levam o profissional a concluir que um problema existe. ${ }^{5}$

A fase dos Diagnósticos de Enfermagem (DE) é imprescindível para um satisfatório direcionamento da SAE. Os DE proporcionam um método útil para organizar o conhecimento, e consistem em uma etapa que para ser realizada necessita do Histórico de Enfermagem e do Exame Físico que é realizado diariamente por meio de técnicas de inspeção, palpação, percussão e ausculta.

$\mathrm{Na}$ área de Saúde da Criança e do Adolescente, chama atenção a falta de consenso acerca dos dados que devem ser coletados pelo enfermeiro junto às crianças e aos adolescentes, para gerar informação, a qual possa subsidiar a tomada de decisão clínica e descrever a contribuição da Enfermagem. ${ }^{7}$

A implementação efetiva da SAE conduz à melhoria da qualidade dos cuidados de saúde da criança e do adolescente, além de estimular a construção de conhecimentos teóricos e científicos com base na melhor prática clínica, e auxiliar na elaboração de protocolos que sejam efetivos à prática da enfermagem pediátrica. ${ }^{8}$

Apesar do número relevante de pesquisas recentes relacionadas à SAE no Brasil, especificamente aos $\mathrm{DE}$, percebe-se que a importância dessa temática na enfermagem pediátrica ainda é pouco elementar ${ }^{9}$, e muitas unidades de saúde encontram barreiras por parte dos profissionais de enfermagem para implementarem a SAE, a exemplo da UTIP referenciada neste estudo.

Diante desse contexto surgiu a motivação para a realização desta pesquisa que teve como pergunta norteadora: Quais os diagnósticos de enfermagem mais frequentes em uma UTIP?

A identificação dos DE não expõe apenas as consequências geradas pelos processos patológicos da criança e do adolescente, mas também aquelas geradas pelos agentes que os permeiam, sabido que uma assistência prestada de maneira imprópria pelos profissionais de saúde, refletirá no surgimento de diagnósticos mais graves.

Frente a essas considerações, este estudo teve como objetivo identificar os dez diagnósticos de enfermagem mais frequentes em pacientes internados em uma Unidade de Terapia Intensiva Pediátrica.

\section{MÉTODO}

Estudo transversal, descritivo, exploratório e quantitativo realizado em uma UTIP de um Hospital Público em Campina Grande-PB. A instituição é apresentada como referência no Estado, sendo o segundo maior hospital do Norte-Nordeste em atendimento a casos de alta complexidade a pacientes assistidos pelo Sistema Único de Saúde (SUS).

A população do estudo foi constituída por pacientes internados na UTI pediátrica, que atende crianças a partir dos 29 dias de nascido até adolescentes com 17 anos 11 meses e 29 dias. A amostra, composta por 30 pacientes, foi definida de forma não probabilística, por conveniência.

O estudo foi aprovado pelo Comitê de Ética em Pesquisa da Universidade Estadual da Paraíba, registrado sob parecer $\mathrm{n}^{\circ}$ 0277.0.133.000-12, atendendo às exigências da Resolução 466/2012 do Conselho Nacional de Saúde ${ }^{10}$, para pesquisas com seres humanos. A coleta de dados aconteceu durante os meses de janeiro e fevereiro de 2013. A seleção das crianças e dos adolescentes foi realizada pela pesquisadora a partir dos prontuários, em que se buscou informações sobre o histórico da doença das crianças e adolescentes, o tempo e a evolução da internação e os relacionamentos familiares, por exemplo. De posse 


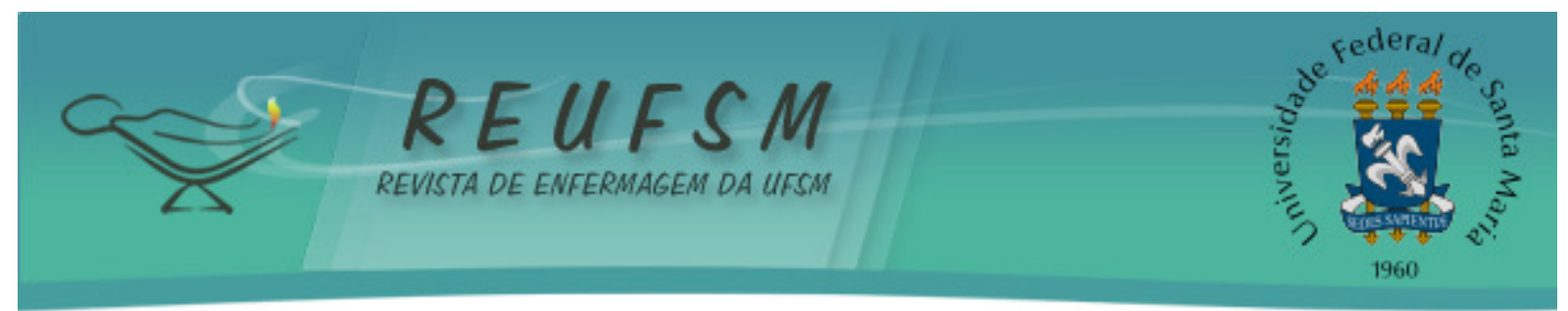

do perfil do paciente, a pesquisadora se apresentava à criança ou ao adolescente e após serem esclarecidos os objetivos do estudo e do Termo de Consentimento Livre e Esclarecido (TCLE) ser assinado pelo responsável legal, era iniciada uma entrevista semiestruturada utilizando-se o Roteiro de Histórico de Enfermagem específico para UTI ${ }^{11}$, no qual foram realizados alguns acréscimos de tópicos como as enfermidades na infância, o histórico de imunizações, duração da amamentação, desenvolvimento geral da criança (físico, motor, cognitivo, padrão de sono), no intuito de tornar o instrumento mais específico à faixa etária da população.

Construído o histórico de enfermagem, que promove um levantamento de informações sobre a vida e saúde do paciente sendo subsídios para a definição do diagnóstico de enfermagem e posterior prescrição e evolução de enfermagem, o exame físico era realizado e, complementarmente, informações eram coletadas nos prontuários, como valores de exames laboratoriais e prescrições medicamentosas.

Os dados coletados foram organizados e analisados em planilhas do software EXCEL (Microsoft Office 2007) com estatística descritiva e por meio de frequência absoluta e relativa, objetivando identificar os problemas, necessidades e reações humanas do paciente que contemplam o âmbito fisiológico, psicossocial, econômico e cultural do ser humano. NANDA. ${ }^{6}$

Para a definição dos diagnósticos de enfermagem, foi utilizada a Taxonomia II da

\section{RESULTADOS E DISCUSSÃO}

Os resultados encontrados referem-se a uma amostra de 30 pacientes $(n=30)$, com idade entre quatro meses e 17 anos, sendo que ambos os sexos tiveram a mesma proporção na amostra, $50 \%$ do sexo feminino e $50 \%$ do masculino. Quanto aos principais diagnósticos médicos desses pacientes, seis $(20,00 \%)$ se referiam ao sistema nervoso, seis $(20,00 \%)$ a traumatismos crânio encefálicos, quatro $(13,33 \%)$ ao sistema respiratório, três $(10,00 \%)$ ao sistema digestivo, dois $(6,67 \%)$ ao sistema músculo/esquelético, dois $(6,67 \%)$ ao sistema cardiovascular, e sete $(23,33 \%)$ a outros diagnósticos.

Adotando como base os dez diagnósticos de enfermagem mais encontrados na UTIP, o domínio mais presente foi o de número 11 (Segurança/proteção) com cinco DE e cuja definição é "estar livre de perigo, lesão física ou dano ao sistema imunológico; conservação contra perdas e proteção da segurança e da ausência de perigos". ${ }^{6} \mathrm{Em}$ segundo lugar, o domínio três (Eliminação e troca) - "secreção e excreção de produtos residuais do organismo" 6 - com dois DE. E por fim, os seguintes domínios com um diagnóstico: o dois (Nutrição) - "atividades de ingerir, assimilar e usar nutrientes com fins de manter e reparar tecidos e produzir energia"6, o quatro (Atividade/ repouso) "produção, conservação, gasto ou equilíbrio de recursos energéticos"6, e o 12 (Conforto) "sensação de bem-estar ou tranquilidade mental, física ou social". ${ }^{6}$ A tabela 1 expõe os domínios acima enunciados junto às classes dos dez DE mais encontrados. 


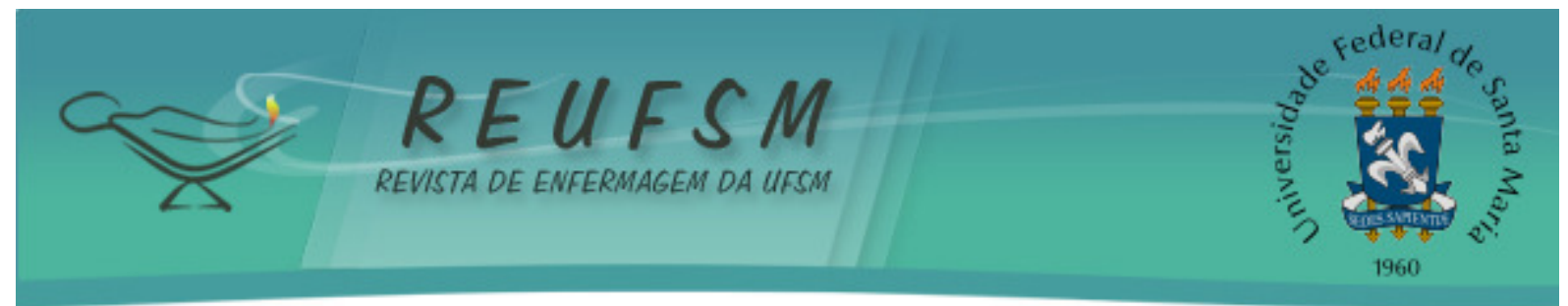

Tabela 1- Domínios e classes dos diagnósticos de enfermagem mais frequentes em uma Unidade de Terapia Intensiva Pediátrica. Campina Grande, PB, Brasil, 2013.

\begin{tabular}{|c|c|c|}
\hline DOMINIO & CLASSE & $\begin{array}{c}\text { CONCEITO DO DIAGNOSTTICO DE } \\
\text { ENFERMAGEM }\end{array}$ \\
\hline $\begin{array}{c}11 \\
\text { Segurança/ } \\
\text { proteção }\end{array}$ & $\begin{array}{c}1 \text { (Infecção) } \\
2 \text { (Lesão física) } \\
2 \text { (Lesão física) } \\
2 \text { (Lesão física) } \\
2 \text { (Lesão física) }\end{array}$ & $\begin{array}{c}\text { - Risco de Infecção } \\
\text { - Desobstrução Ineficaz de vias aéreas } \\
\text { - Risco de Integridade da pele prejudicada } \\
\text { - Risco de Trauma Vascular } \\
\text { - Integridade da pele prejudicada }\end{array}$ \\
\hline $\begin{array}{c}3 \\
\text { Eliminação e troca }\end{array}$ & $\begin{array}{l}2 \text { (Função gastrintestinal) } \\
2 \text { (Função gastrintestinal) }\end{array}$ & $\begin{array}{l}\text { - Motilidade gastrintestinal disfuncional } \\
\bullet \quad \text { Constipação }\end{array}$ \\
\hline $\begin{array}{c}2 \\
\text { Nutrição }\end{array}$ & 5 (Hidratação) & - Risco de desequilíbrio eletrolítico \\
\hline $\begin{array}{c}4 \\
\text { Atividade/ } \\
\text { repouso }\end{array}$ & $\begin{array}{c}4 \text { (Respostas } \\
\text { cardiovasculares/ } \\
\text { pulmonares) }\end{array}$ & - Ventilação espontânea prejudicada \\
\hline $\begin{array}{c}12 \\
\text { Conforto }\end{array}$ & 1 (Conforto físico) & - Dor aguda \\
\hline
\end{tabular}

O gráfico 1 apresenta, por ordem de maior frequência em aparecimento, os DE mais freqüentes encontrados nesta pesquisa que foi realizada em uma UTIP de um hospital público do município de Campina Grande-PB.

Gráfico 1 - Diagnósticos de enfermagem mais frequentes em uma Unidade de Terapia Intensiva Pediátrica. Campina Grande, PB, Brasil, 2013.

\section{Diagnósticos de Enfermagem}

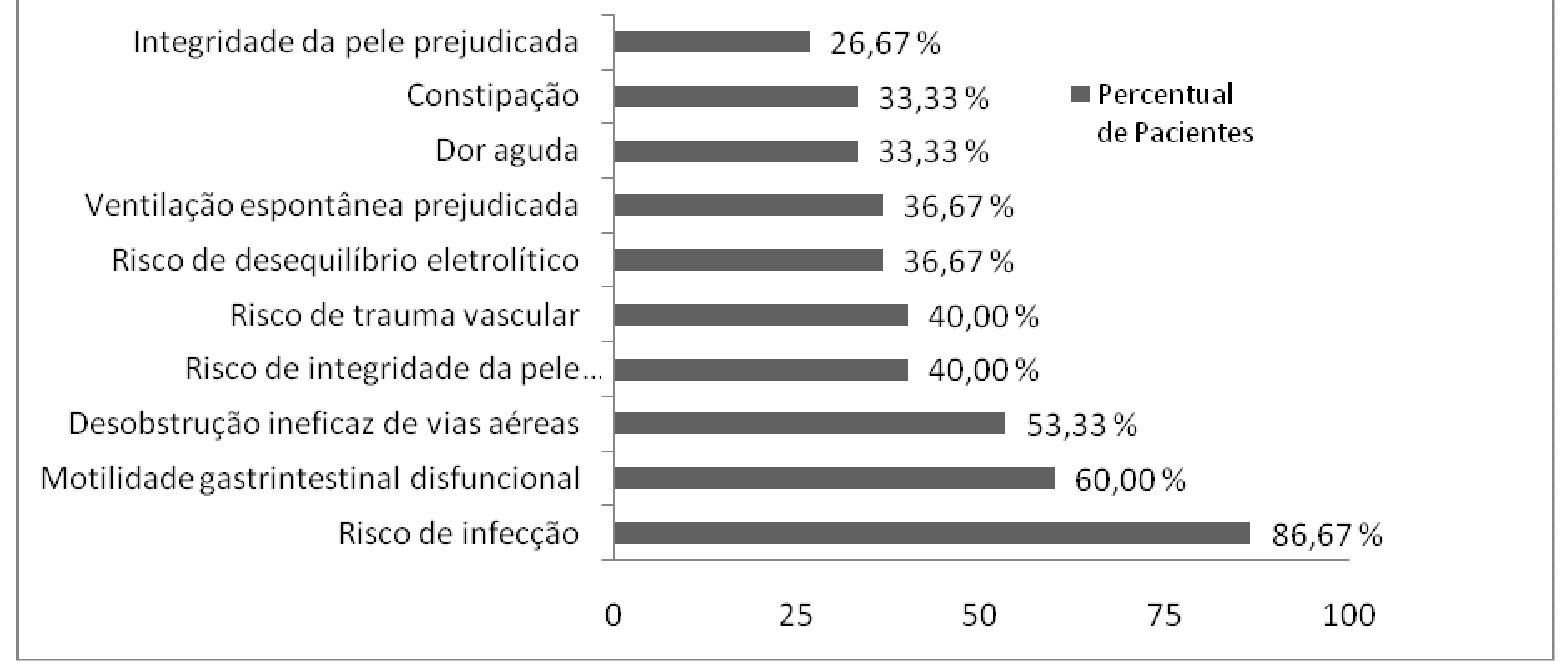

No gráfico 1 observa-se que o DE mais encontrado foi "Risco de infecção", 86,67\%, cuja definição é risco aumentado de ser invadido por organismos patogênicos. ${ }^{6}$ Tal diagnóstico está incluído no domínio 11 e classe 1 e também foi um dos mais vistos em outros estudos $^{12-13}, 96,8 \%$ e $100 \%$ dos pacientes, respectivamente. Para esse DE os principais 


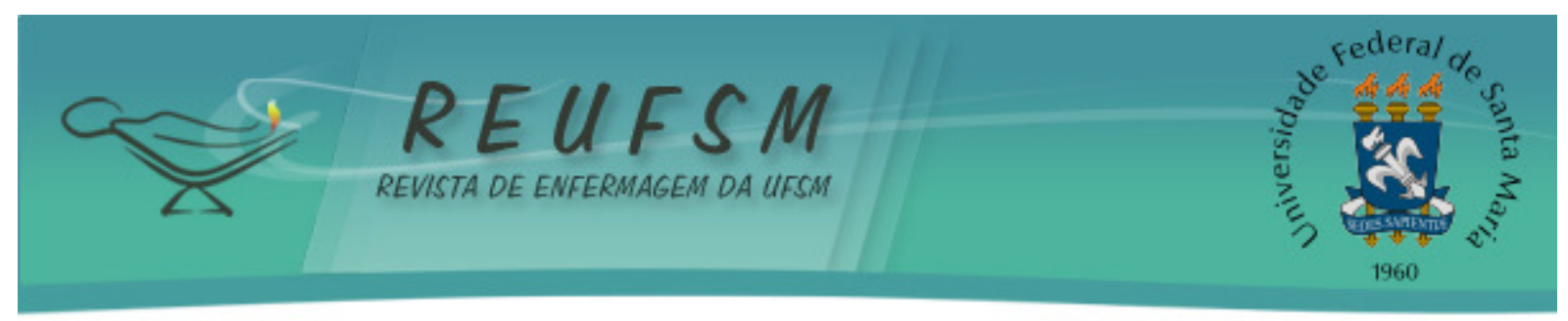

fatores de risco foram: procedimentos invasivos, alterações laboratoriais, incisão cirúrgica, dessecção venosa e úlcera por pressão.

O Risco de Infecção pode ser influenciado pelo número de profissionais que lida diretamente com o paciente, a quantidade e o tipo de procedimentos invasivos, a forma de assistência que é dispensada e o tempo de hospitalização. Esse diagnóstico descreve um estado que pode comprometer a recuperação de pacientes internados em uma UTI ${ }^{13}$, por isso é importante que o enfermeiro realize o DE e elabore um plano de cuidados, pois assim, sua equipe estará prestando uma assistência de enfermagem com medidas eficazes no controle de infecção.

Dentre os dispositivos invasivos mais encontrados nos pacientes estavam: punção venosa periférica $(60 \%)$, sonda vesical de demora $(33,33 \%)$, tubo orotraqueal $(23,33 \%)$, punção venosa central $(23,33 \%)$, drenos em geral $(20 \%)$ e traqueostomia $(10 \%)$. Esses dispositivos são fundamentais à manutenção da vida do paciente, mas devem ser monitorados e higienizados para que não se tornem um meio para proliferação de microorganismos. ${ }^{13}$

0 segundo diagnóstico de enfermagem mais encontrado foi "Motilidade gastrintestinal disfuncional" - atividade peristáltica aumentada, diminuída, ineficaz ou ausente no sistema gastrintestinal ${ }^{6}$ - com $60 \%$ de frequência da amostra. 0 mesmo pertence ao domínio 3 e à classe 2 . As características definidoras identificadas para esse diagnóstico foram: sons intestinais ausentes e hiperativos, abdome globoso, distensão abdominal, náusea, ausência de evacuações, evacuações frequentes ao dia, vômito, esvaziamento gástrico acelerado e dor abdominal. Estas estavam relacionadas à ansiedade, ao ambiente hospitalar, agentes farmacêuticos, procedimento cirúrgico, alimentação por sonda, imobilidade, disfunção neuromuscular, hemorragia digestiva baixa e intolerância à lactose.

Quando internados alguns indivíduos têm seus hábitos alimentares modificados o que os predispõem a um estado nutricional alterado. ${ }^{3}$ Motilidade gastrintestinal disfuncional também foi encontrado em $45 \%$ dos pacientes avaliados em uma pesquisa ${ }^{3}$ sobre SAE em uma Unidade de Terapia Intensiva.

"Desobstrução ineficaz de vias aéreas" - domínio 11, classe 2 - definido como a incapacidade de eliminar secreções ou obstruções do trato respiratório para manter uma via aérea desobstruída ${ }^{6}$, foi o terceiro DE mais encontrado, sendo identificado em $53,33 \%$ dos pacientes.

As características definidoras encontradas: ruídos adventícios respiratórios (roncos, sibilos), tosse produtiva, aumento da frequência respiratória, dispneia, traqueostomia e dificuldade de expectoração. Em 81,25\% dos pacientes com desobstrução ineficaz de vias aéreas foi observada a presença de ruídos adventícios na ausculta pulmonar e fatores relacionados que os impediam de expectorar as secreções brônquicas e o muco excessivo como presença de via aérea artificial (25\%) e disfunção neuromuscular. Nos pacientes traqueostomizados e na presença de vias aéreas artificiais, percebeu-se um aumento da frequência respiratória. Esse achado clínico pode surgir como uma maneira do organismo alcançar os níveis de oxigênio necessários ao bom funcionamento metabólico do corpo. ${ }^{14}$

Se a desobstrução ineficaz de vias aéreas não for adequadamente tratada, poderá levar a outras alterações como, por exemplo, padrão respiratório ineficaz ou troca de gases prejudicada, o que acarretaria em sofrimento respiratório e até morte. ${ }^{14}$

O diagnóstico "Risco de integridade da pele prejudicada" - risco de a pele ser alterada de forma adversa ${ }^{6}$ - apareceu em quarto lugar com $40 \%$. Ele faz parte do domínio 11 e da classe 2.

O Risco de integridade da pele prejudicada apresentou os seguintes fatores de risco: pruridos, fatores mecânicos (contenção), fatores de desenvolvimento, procedimentos invasivos, imobilização física, sobrepeso, sedação contínua e evacuações frequentes. Dos pacientes com esse diagnóstico, 66,67\%, estavam contidos ao leito. 


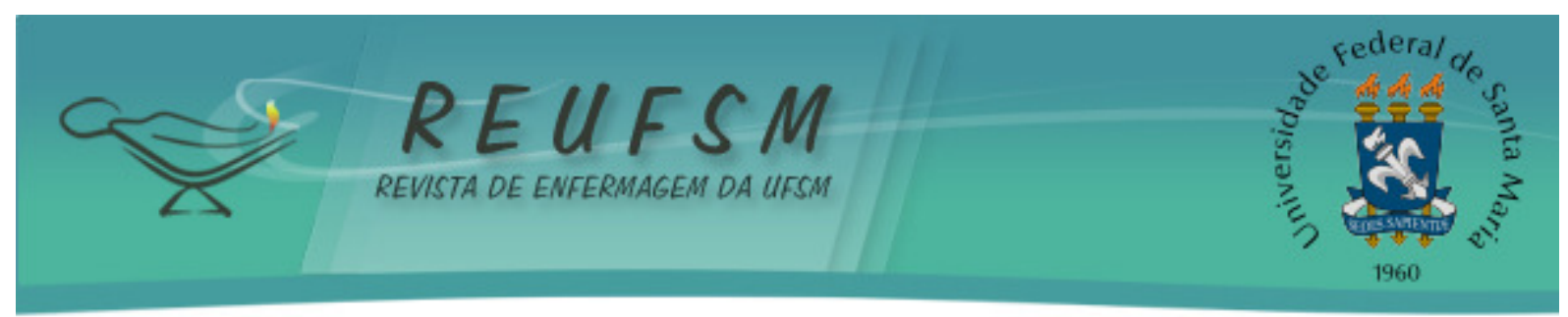

A identificação deste diagnóstico contribui para o planejamento das intervenções de enfermagem necessárias, no intuito de prevenir e reduzir as incapacidades e recuperar a saúde. ${ }^{15}$

Em quinto lugar, em $40 \%$ dos diagnósticos levantados dos 30 pacientes, apareceu o diagnóstico "Risco de trauma vascular", pertencente ao domínio 11, classe 2, o qual é caracterizado pelo risco de dano à veia e tecidos ao redor, relacionado à presença de cateter e/ou soluções infundidas. ${ }^{6}$

Os principais fatores de risco para o diagnóstico Risco de trauma vascular foram: punções periféricas, acesso venoso central, dissecção venosa, cateter com duplo lúmen em femoral, tipo de cateter, local da inserção. Todos os pacientes (100\%) com esse diagnóstico estavam com algum tipo de cateter.

Assim como no diagnóstico Risco de infecção, aqui, os procedimentos invasivos sobrepujaram os potenciais problemas à saúde dos pacientes. No caso deste estudo, em uma UTIP, o risco de trauma vascular torna-se mais frequente em virtude do fato de, dependendo do local da inserção do cateter, o incômodo gerado na criança pode ser bem maior que em um adulto.

O diagnóstico de risco para traumas potenciais ocorrem nos vasos sanguíneos, áreas adjacentes e apresentam efeitos locais ou sistêmicos em decorrência do processo de punção vascular. ${ }^{16}$ "A formação profissional e/ou a aquisição de habilidades por pessoas leigas são fundamentais para minimizar danos decorrentes do processo de punção vascular". ${ }^{16}$

0 "Risco de desequilíbrio eletrolítico" (domínio 2 e classe 5) - risco de mudança nos níveis eletrolíticos séricos capaz de comprometer à saúde ${ }^{6}$ - veio em sexto lugar com $36,67 \%$.

Segundo o próprio conceito, esse diagnóstico envolve desequilíbrios relacionados à perda ou ganho excessivo de água e demais eletrólitos no organismo, capaz de prejudicar a saúde humana. Seus fatores de risco: drenagem torácica e intracraniana, níveis alterados de sódio, potássio, cálcio, ureia e creatinina, medicamentos diuréticos, frequentes evacuações ao dia, disfunção renal, vômitos e poliúria, os quais podem representar uma dificuldade para que o corpo desempenhe seu metabolismo funcional normalmente. Em $54,55 \%$ dos pacientes com risco de desequilíbrio eletrolítico, houve alterações nos níveis laboratoriais de alguns eletrólitos (sódio, potássio, ureia, cálcio e creatinina).

$\mathrm{Na}$ sétima posição, com $36,67 \%$, está o diagnóstico “Ventilação espontânea prejudicada", domínio 4, classe 4 e definição - reservas de energia diminuídas, resultando em uma incapacidade do indivíduo de manter respiração adequada para sustentação da vida. ${ }^{6}$

Dentre as principais características definidoras para Ventilação espontânea prejudicada estavam: dispneia, alterações gasométricas, frequência cardíaca aumentada e acidose metabólica e respiratória. Para contextualizar essas características seguiram os fatores: distrofia muscular, atelectasia, insuficiência respiratória aguda e derrame pleural.

A ventilação disfuncional é definida como o funcionamento anormal ou incompleto do movimento do ar para dentro e para fora dos pulmões com certo padrão e ritmo respiratório, profundidade de inspiração e força de expiração. ${ }^{3}$ Dos pacientes com ventilação espontânea prejudicada, 63,64\% estavam com alterações nos valores da gasometria e $45,45 \%$ em sedação contínua.

Autores identificaram "Ventilação espontânea prejudicada" em 95\% dos pacientes e afirmaram que esse diagnóstico se relaciona à mecânica da ventilação e não aos processos de troca gasosa e transporte de oxigênio/dióxido de carbono. ${ }^{3}$

Em oitavo lugar está "Dor aguda" (33,33\%) - experiência sensorial e emocional desagradável que surge de lesão tissular real, potencial ou descrita em termos de tal 


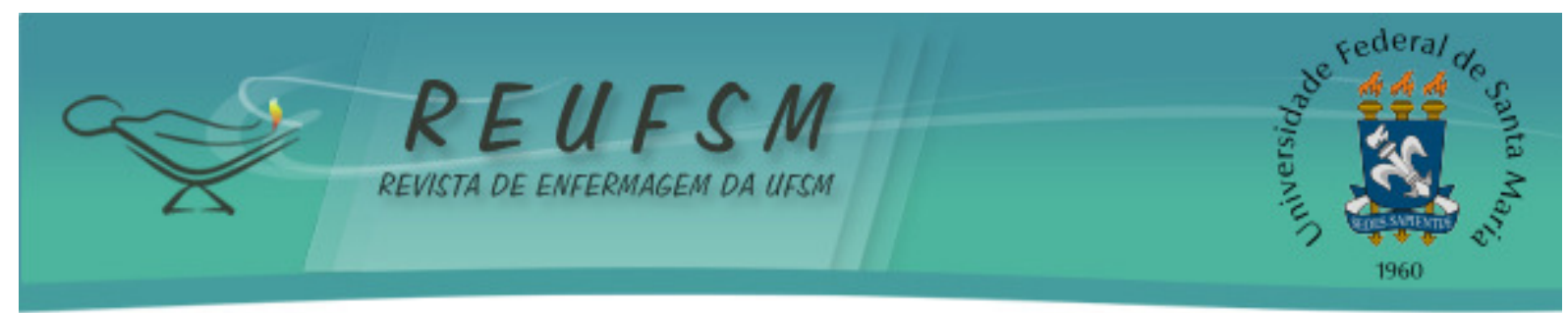

lesão; ${ }^{17}$ início súbito ou lento, de intensidade leve a intensa, com término antecipado ou previsível e duração de menos de seis meses ${ }^{6}$ - pertencente ao domínio 12 e à classe 1.

A dor é uma das principais causas de sofrimento humano, comprometendo a qualidade de vida das pessoas e refletindo no seu estado físico e psicossocial. Ela envolve vários componentes sensoriais, afetivos e cognitivos, sociais e comportamentais. ${ }^{18}$ As características que definiram a Dor aguda foram: relato verbal, expressões faciais, choro intenso, dificuldade para dormir, agitação e posição para evitar a dor. Dentre os principais fatores relacionados a esse DE estão os procedimentos invasivos e cirúrgicos; e $80 \%$ dos pacientes com dor aguda relataram verbalmente o incômodo.

O diagnóstico “Constipação” apareceu, com 33,33\%, em nono lugar. É definido como a diminuição na frequência normal de evacuação, acompanhada por passagem de fezes difícil ou incompleta e/ou eliminação de fezes excessivamente duras e secas. ${ }^{6}$ Faz parte do domínio 3 e da classe 2. Para esse diagnóstico os pacientes apresentaram: distensão abdominal, náusea, ruídos intestinais hiperativos e hipoativos, frequência diminuída, abdome globoso, abdome depressível e flácido e incapacidade de eliminar as fezes. Muitos desses sinais e sintomas estavam relacionados a alguns dos seguintes fatores: agentes farmacêuticos, motilidade do trato gastrintestinal diminuída, mudanças nos alimentos usuais, atividade física insuficiente, desidratação e ingestão insuficiente de fibras e líquidos.

Um estudo ${ }^{12}$ similar sobre DE na UTI encontrou um percentual próximo ao deste estudo para o diagnóstico de Constipação, 34,3\% dos pacientes. Os hábitos alimentares e o estado nutricional dos pacientes internados em uma UTI são influenciados por fatores relacionados como: má absorção gastrintestinal e desidratação. ${ }^{13}$

"Integridade da pele prejudicada" $(26,67 \%)$ foi o décimo DE mais encontrado. Esse diagnóstico está incluído no domínio 11 da classe 2, e leva em consideração apenas as lesões cutâneas, ou seja, na epiderme e derme, ocasionando assim falhas na primeira linha de defesa do organismo.

Alguns autores observaram em seus estudos sobre DE em UTI, a Integridade da pele prejudicada em $10 \%^{3}$ e $88,3 \%^{13}$ dos pacientes internados.

A Integridade da pele prejudicada caracterizou-se principalmente pelo rompimento da superfície da pele, seja em decorrência de um trauma ou pelo desenvolvimento de úlceras por pressão, sendo que apenas 01 (um) paciente da amostra apresentou úlcera por pressão. Fatores de desenvolvimento e mecânicos, além de incisões cirúrgicas relacionaram-se a esse DE.

\section{CONCLUSÕES}

A SAE constitui um caminho a ser utilizado pelos enfermeiros para a realização das suas ações, como a identificação das respostas dos pacientes aos problemas de saúde e aos processos vitais que exigem intervenções.

A identificação dos diagnósticos de enfermagem permite que a assistência prestada seja direcionada e específica aos reais e potenciais problemas de saúde do sujeito a ser cuidado. Em crianças e adolescentes com estado de saúde crítico, como os que se encontram internados em uma UTI, é essencial que os achados clínicos sejam identificados corretamente para que o enfermeiro possa desenvolver ações assertivamente.

Os resultados deste estudo podem contribuir para implantação da SAE na UTIP em questão, além de fornecer informações para outras pesquisas acerca dos dados que devem ser coletados pelos enfermeiros na assistência à criança e ao adolescente. Porém, é necessário mais estudos sobre a temática para que outros DE sejam identificados, possibilitando uma avaliação mais abrangente da eficácia da sistematização da assistência de enfermagem. 


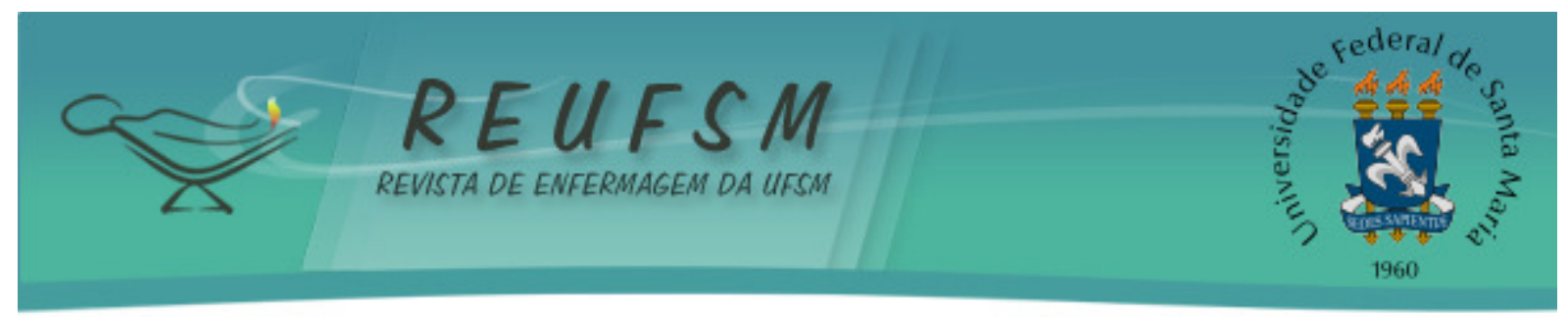

\section{REFERÊNCIAS}

1. Molina RCM, Varela PRL, Castilho AS, Bercini LO, Marcon SS. Presença da família nas unidades de terapia intensiva pediátrica e neonatal: visão da equipe multidisciplinar. Esc Anna Nery Rev Enferm. 2007;11(3):437-44.

2. Inoue KC, Matsuda LM. Dimensionamento de pessoal de enfermagem em Unidade de Terapia Intensiva para adultos. Acta Paul Enferm. 2010;23(3):379-84.

3. Truppel TC, Meier MJ, Riciana CC, Peruzzo SA, Crozeta K. Sistematização da Assistência de Enfermagem em Unidade de Terapia Intensiva. Rev Bras Enferm. 2009;62(2):221-7.

4. Adamy EK, Tosatti M. Sistematização da Assistência de Enfermagem no período perioperatório: visão da equipe de enfermagem. Rev Enferm UFSM [Internet] 2012 maio/ago [acesso em 2014 out 24];2(2):300-10. Disponível em: http://cascavel.ufsm.br/revistas/ojs-2.2.2/index.php/reufsm/article/view/5054/3754.

5. Tannure MC, Gonçalves AMP. SAE: Sistematização da Assistência de Enfermagem. São Paulo: Guanabara Koogan; 2008. 168 p.

6. Diagnósticos de Enfermagem da NANDA 2009-2011. Porto Alegre: Artmed; 2010.

7. Marques DKA, Souza GLL, Silva AB, Silva AF, Nóbrega MML. Conjunto Internacional de Dados Mínimos de Enfermagem: estudo comparativo com instrumentos de uma clinica pediátrica. Rev Bras Enferm. 2014;67(4):588-93.

8. Lima APS, Chianca TCM, Tannure MC. Avaliação da assistência de enfermagem utilizando indicadores gerados por um software. Rev Latinoam Enferm. 2015;23(2):234-41.

9. Nery IS, Santos AG, Sampaio MRFB. Dificuldades para a implantação sistematização da assistência de enfermagem em maternidades. Enfermagem em Foco [internet]. 2013 [acesso em 2014 set 18];4(1):11-4. Disponível em: http://revista.portalcofen.gov.br/index.php/enfermagem/article/viewFile/494/184.

10. Ministério da Saúde. Conselho Nacional de Saúde. Resolução CNS n466, de 12 de dezembro de 2012. Aprova diretrizes e normas regulamentadoras de pesquisas envolvendo seres humanos. Brasília; 2012 [acesso em 2013 ago 30]. Disponível em: http://conselho.saude.gov.br/resolucoes/2012/Reso466.pdf.

11. Amante LN, Rossetto AP, Schneider DG. Sistematização da Assistência de Enfermagem em Unidade de Terapia Intensiva sustentada pela teoria de Wanda Horta. Rev Esc Enferm USP. 2009;43(1):54-64.

12. Pasini D, Alvim I, Kanda L, Mendes RSP, Cruz DALM. Diagnósticos de enfermagem de pacientes internados em unidades de terapia intensiva. Rev Esc Enf USP. 1996;30(3):50118.

13. Salomé GM. Diagnóstico de enfermagem dos pacientes internados em uma Unidade de Terapia Intensiva. Saúde Coletiva [Internet]. 2011 [acesso em 2013 jun 24];8(47):24-8. Disponível em: http: / /www.redalyc.org/articulo.oa?id=84217101006.

14. Martins I, Gutiérrez MGR. Intervenções de enfermagem para o diagnóstico de enfermagem Desobstrução ineficaz de vias aéreas. Acta Paul Enferm. 2005;18(2):143-9.

15. Moreira RP, Oliveira ARS, Costa AGS, Cavalcante TF, Guedes NG, Araujo TL. Risco de integridade da pele prejudicada em pacientes acometidos por acidente vascular 


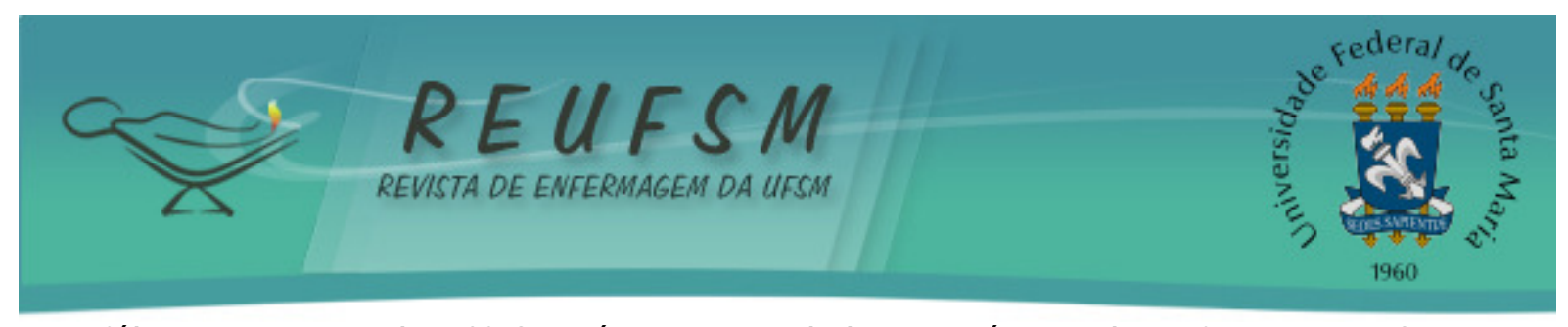

encefálico. In: Anais do $10^{\circ}$ Simpósio Nacional de Diagnóstico de Enfermagem (SINADEn); 2010 ago 4-7, Brasília. Brasília: ABEn-Seção Distrito Federal; 2010. p. 247-50.

16. Arreguy-Sena C, Carvalho EC. Risco para trauma vascular: proposta do diagnóstico e validação por peritos. Rev Bras Enferm. 2009;62(1):71-8.

17. International Association for the Study of Pain (IASP). Pain terms: list with definitions and notes on usage: recommended by the Subcommitee on Taxonomy [Internet]. [acesso em 2014 fev 10]. Disponível em: http://www.iasppain.org/Taxonomy?navltemNumber=576\#Pain.

18. Rigotti MA, Ferreira AM. Intervenções de enfermagem ao paciente com dor. Arq Ciênc Saúde. 2005;12(1):50-4.

Data de recebimento: $15 / 12 / 2014$

Data de aceite: $11 / 08 / 2015$

Contato do autor responsável: Danila Maria Batista Guedes

Endereço postal: Av. Dr. Enéas de Carvalho, 419 - Cerqueira César. Escola de Enfermagem da USP. Departamento de Enfermagem Materno-Infantil e Psiquiátrica. CEP 05403-000, São Paulo, SP, Brasil.

E-mail:danilaguedes@usp.br 